\title{
Bioinformatic Prediction of Gene Ontology Terms of Uncharacterized Proteins from Chromosome 11
}

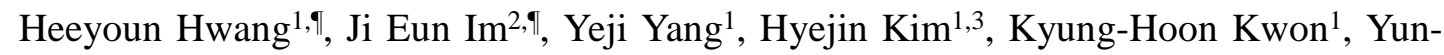
Hee $\mathrm{Kim}^{2,4, *}$, Jin Young Kim${ }^{1, *}$, and Jong Shin Yoo $^{1,3, *}$

${ }^{1}$ Research Center for Bioconvergence Analysis, Korea Basic Science Institute, Cheongju, Republic of Korea, 28119

${ }^{2}$ Division of Convergence Technology, Research Institute of National Cancer Center, Goyang, Republic of Korea, 10408

${ }^{3}$ Graduate School of Analytical Science and Technology, Chungnam National University, Daejeon, Republic of Korea, 34134

${ }^{4}$ Departmentof Cancer Biomedical Science, The National Cancer Center Graduate School of Cancer Science and Policy, Goyang, Republic of Korea, 10408

\section{Contents}

Figure S1. Cscore ${ }^{\mathrm{GO}}$ distribution of 2,413 from 22 PE1 from chromosome 11 predicted GO terms using I-TASSER/COFACTOR. In three class of GO terms such as 'Biological Process' (A), 'Cellular Component' (B) and 'Molecular Function' (C), the GO terms was matched and unmatched against excerpted neXtProt GO terms of gold grade $(30,735)$ and gold/silver grade $(39,909)$ including their corresponding ancestor GO terms downloaded from Quick GO of EMBL-EBI (https://www.ebi.ac.uk). The predicted GO terms of the 22 PE1 from ITASSER/COFACTOR results were extracted from "GOsearchresult_final (...) .csv" files using an in-house program coded in Python (version 3.8). ns: 5.00e-02 < p-value <= 1.00e+00; *: $1.00 \mathrm{e}-02<\mathrm{p}$-value $<=5.00 \mathrm{e}-02 ; * *: 1.00 \mathrm{e}-03<\mathrm{p}$-value $<=1.00 \mathrm{e}-02 ; * * *: 1.00 \mathrm{e}-04<\mathrm{p}<=$ $1.00 \mathrm{e}-03 ; * * * *$ : p-value $<=1.00 \mathrm{e}-04$.

Figure S2. Cscore ${ }^{\mathrm{GO}}$ distribution of $240 \mathrm{GO}$ terms predicted from 22 PE1 using ITASSER/COFACTOR and filtered using a path-to-root and cutting off the general term. In three class of GO terms such as 'Biological Process' (A), 'Cellular Component' (B) and 'Molecular Function' (C), the GO terms was matched and unmatched against excerpted neXtProt GO terms of gold grade $(3,612)$ and gold/silver grade $(4,407)$. The predicted and filtered GO terms of the 22 PE1 were extracted from "GOsearchresult_final (...) .svg" files from ITASSER/COFACTOR results using an in-house program coded in Python (version 3.8). ns: 5.00e-02 < p-value <= 1.00e+00; *: 1.00e-02 < p-value <= 5.00e-02; **: $1.00 \mathrm{e}-03<\mathrm{p}$-value <= $1.00 \mathrm{e}-02 ; * * *: 1.00 \mathrm{e}-04<\mathrm{p}<=1.00 \mathrm{e}-03 ; * * * *$ : p-value $<=1.00 \mathrm{e}-04$.

Figure S3. Cscore ${ }^{\mathrm{GO}}$ distribution of GO terms such as 'Biological Process', 'Cellular Component' and 'Molecular Function' predicted by I-TASSER/COFACTOR from 65 uPE1 in chromosome 11. (A) non-filtered GO terms (B) filtered GO terms (End of GO path) using our pipeline.

Figure S4. Western blot analysis using 293T cells transfected with DDK-tagged plasmid DNA for three indicated uPE1s: CCDC90B (A), C11orf52 (B) and SMAP (C). Data of western blot analysis with anti-CCDC90B(A, upper), anti-C11orf52 (B, upper) anti-SMAP (C, upper), respectively, showed the expression of each uPE1 protein compared to control vector transfection. DDK is detected only in cells expressing CCDC90B (A, bottom), C11orf52 (B, bottom), and SMAP (C, bottom). 


\section{Contents continued}

\section{Supporting excel file}

Table S1. Predicted GO terms from 22 PE1 in chromosome 11 using ITASSER/COFACTOR.

Table S2. Predicted GO term of "Molecular Function" from NX_O00255 (NEM1) using ITASSER/COFACTOR.

Table S3. Predicted and filtered GO term from 22 PE1 in chromosome 11.

Table S4. Predicted GO terms from 65 uPE1 in chromosome 11 using ITASSER/COFACTOR.

Table S5. Predicted and filtered GO terms from 65 uPE1 in chromosome 11.

\section{Supplementary Methods}


Figure S1. Cscore distribution of 2,413 from 22 PE1 from chromosome 11 predicted GO terms using ITASSER/COFACTOR. In three class of GO terms such as 'Biological Process' (A), 'Cellular Component' (B) and 'Molecular Function' (C), the GO terms was matched and unmatched against excerpted neXtProt GO terms of gold grade $(30,735)$ and gold/silver grade $(39,909)$ including their corresponding ancestor GO terms downloaded from Quick GO of EMBL-EBI (https://www.ebi.ac.uk). The predicted GO terms of the 22 PE1 from I-TASSER/COFACTOR results were extracted from "GOsearchresult_final (...) .csv" files using an in-house program coded in Python (version 3.8). ns: 5.00e-02 < p-value <= 1.00e+00; *: $1.00 \mathrm{e}-02<\mathrm{p}$-value $<=$ 5.00e-02; **: $1.00 \mathrm{e}-03<\mathrm{p}$-value $<=1.00 \mathrm{e}-02 ; * * *: 1.00 \mathrm{e}-04<\mathrm{p}<=1.00 \mathrm{e}-03 ; * * * *$ : p-value $<=1.00 \mathrm{e}-04$.

A
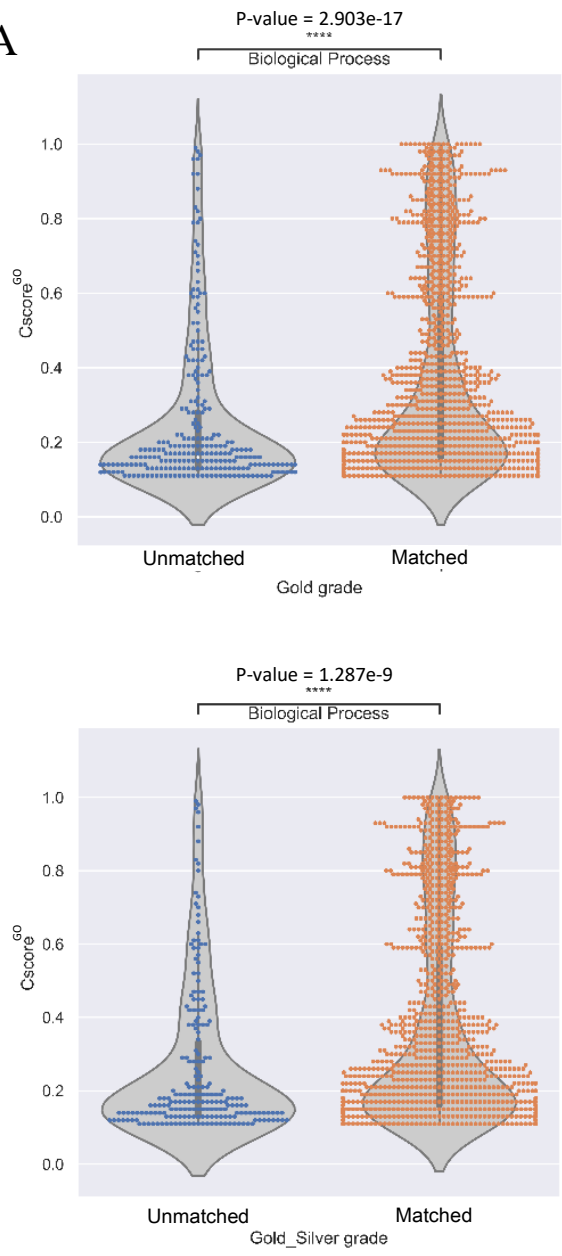

B
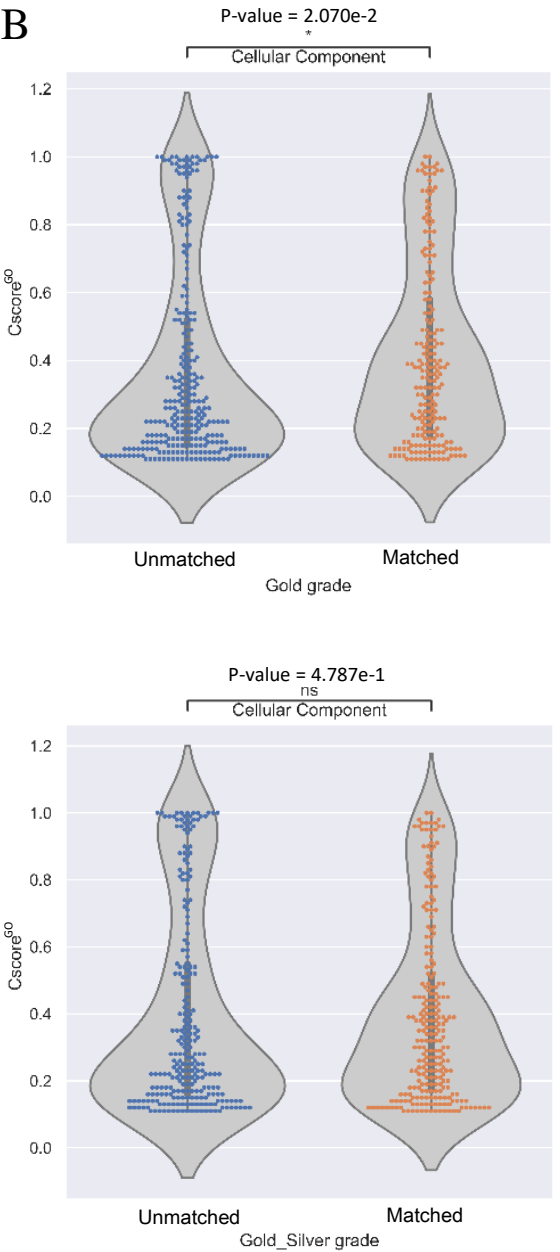
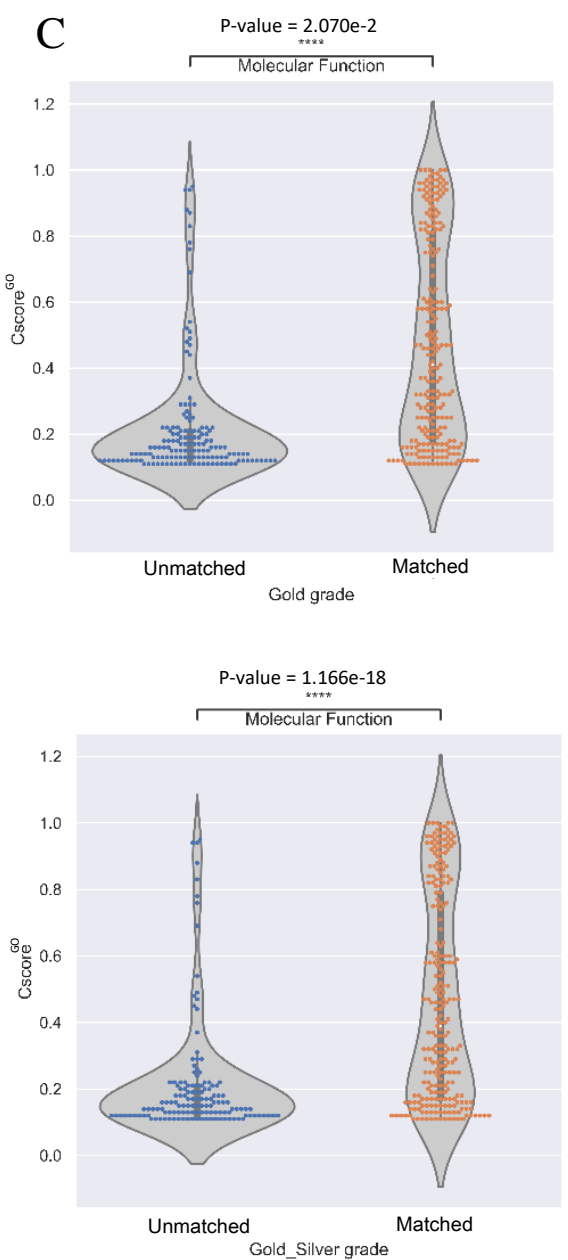
Figure S2. Cscore distribution of $240 \mathrm{GO}$ terms predicted from 22 PE1 using I-TASSER/COFACTOR and filtered using a path-to-root and cutting off the general term. In three class of GO terms such as 'Biological Process' (A), 'Cellular Component' (B) and 'Molecular Function' (C), the GO terms was matched and unmatched against excerpted neXtProt GO terms of gold grade $(3,612)$ and gold/silver grade $(4,407)$. The predicted and filtered GO terms of the 22 PE1 were extracted from "GOsearchresult_final (...).svg" files from ITASSER/COFACTOR results using an in-house program coded in Python (version 3.8). ns: 5.00e-02<p-value <= $1.00 \mathrm{e}+00 ; *$ : $1.00 \mathrm{e}-02<\mathrm{p}$-value <= 5.00e-02; **: $1.00 \mathrm{e}-03<$ p-value < $<1.00 \mathrm{e}-02 ; * * *: 1.00 \mathrm{e}-04<\mathrm{p}<=1.00 \mathrm{e}-03$; $* * * *$ : p-value $<=1.00 \mathrm{e}-04$.

A
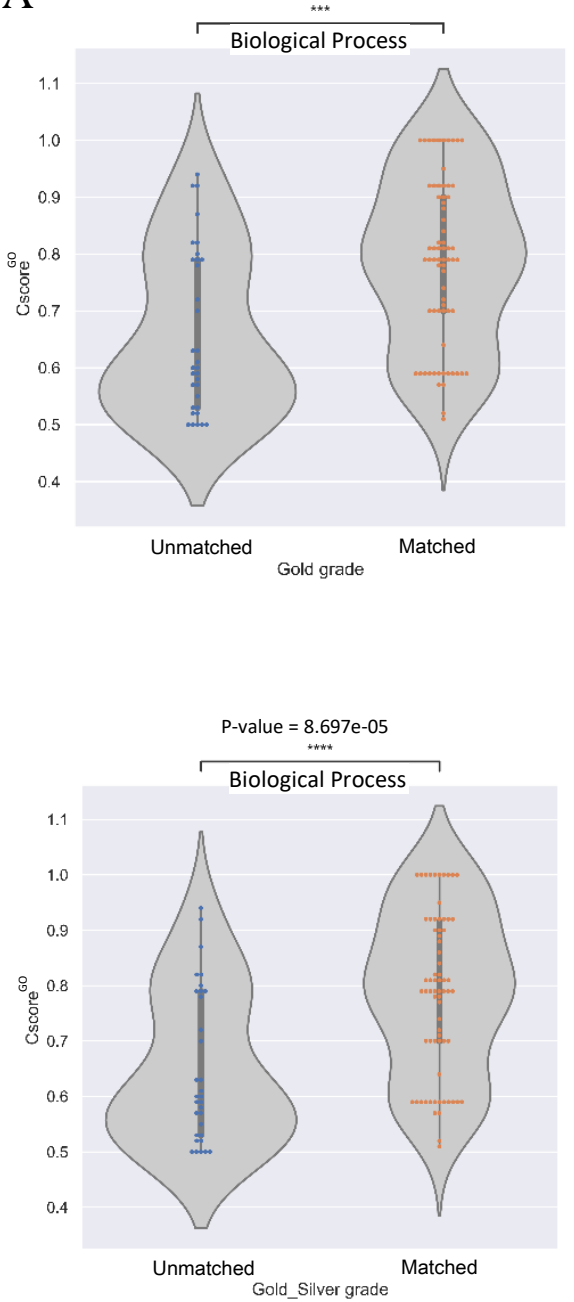

B
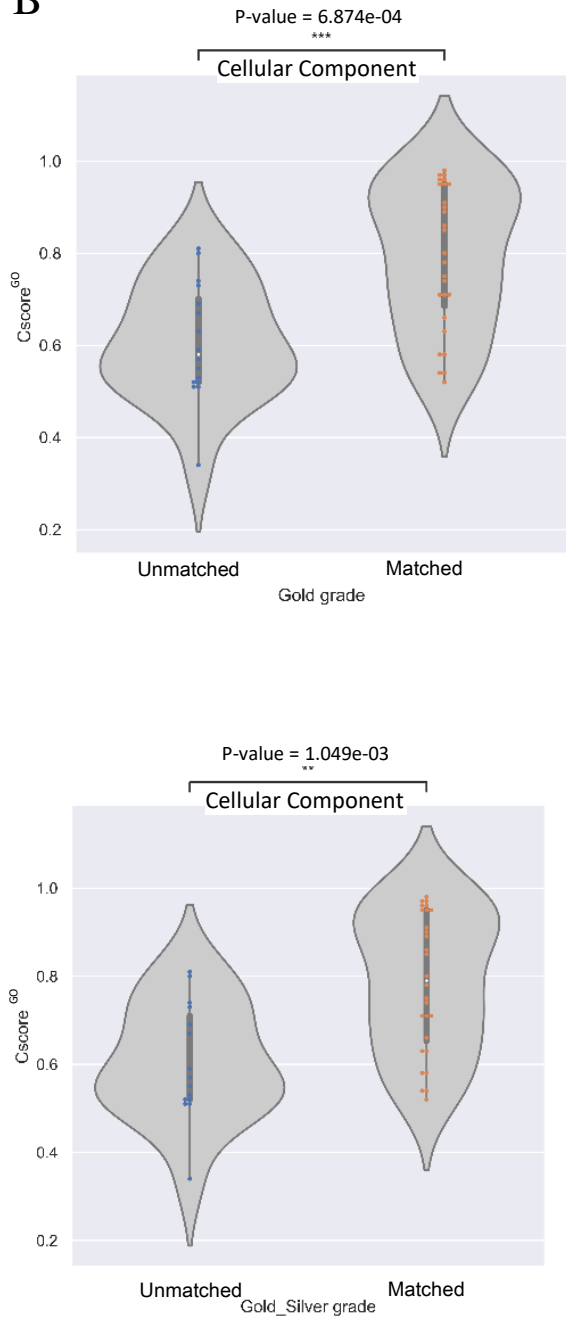
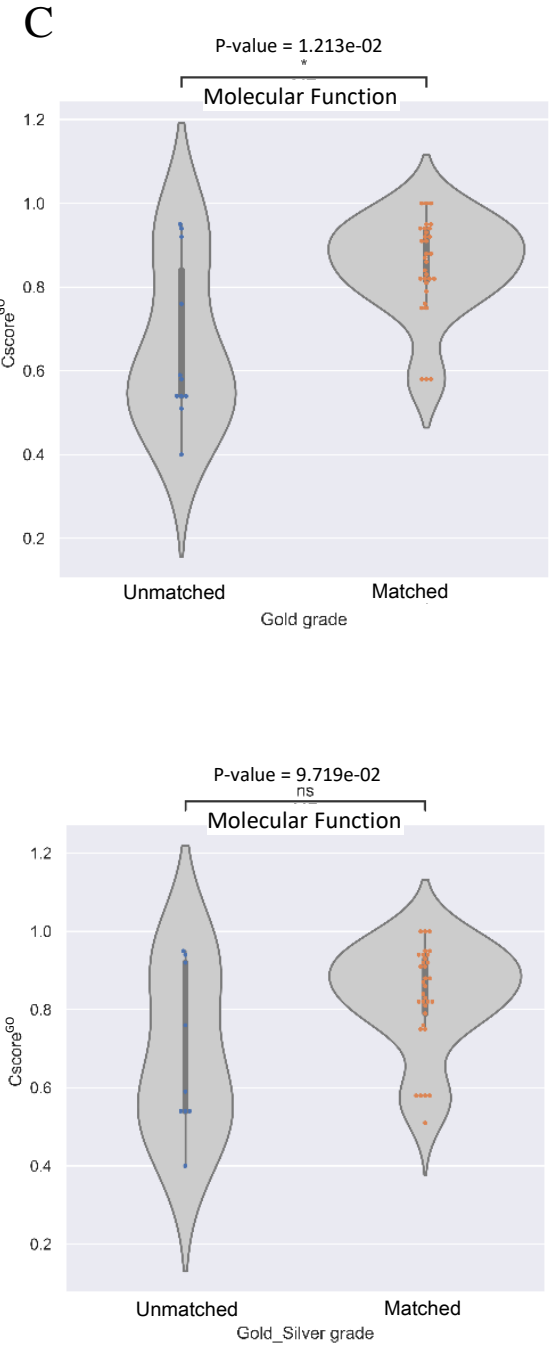
Figure S3. Cscore distribution of GO terms such as 'Biological Process', 'Cellular Component' and 'Molecular Function' predicted by I-TASSER/COFACTOR from 65 uPE1 in chromosome 11. (A) nonfiltered GO terms (B) filtered GO terms (End of GO path) using our pipeline.

A

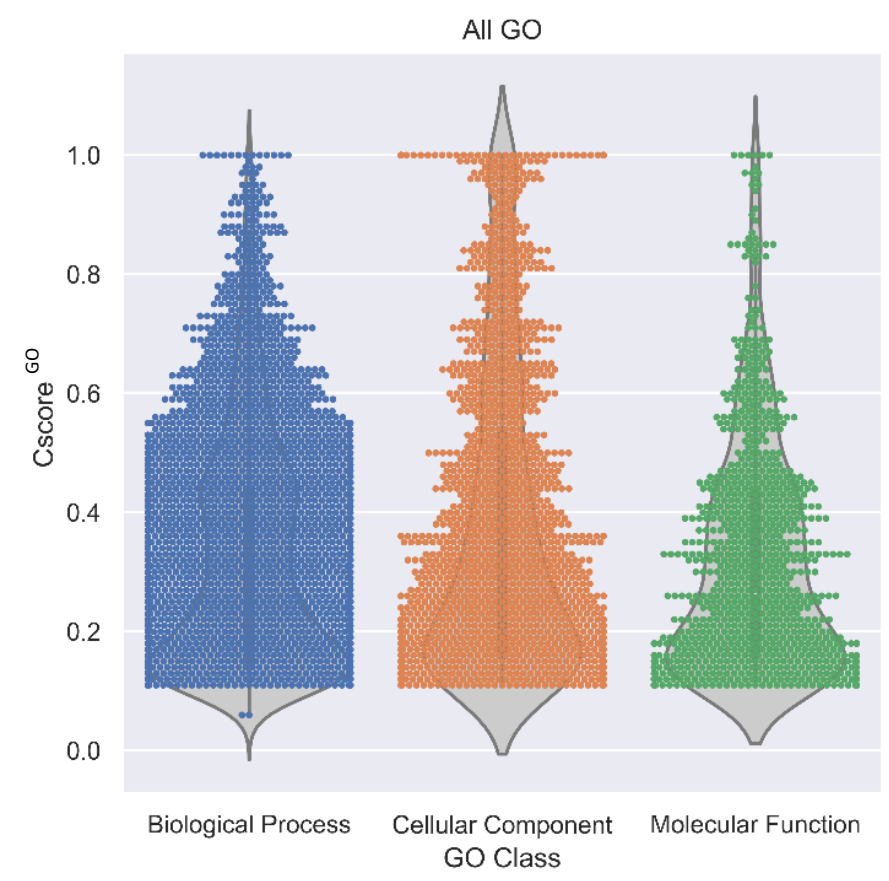

B

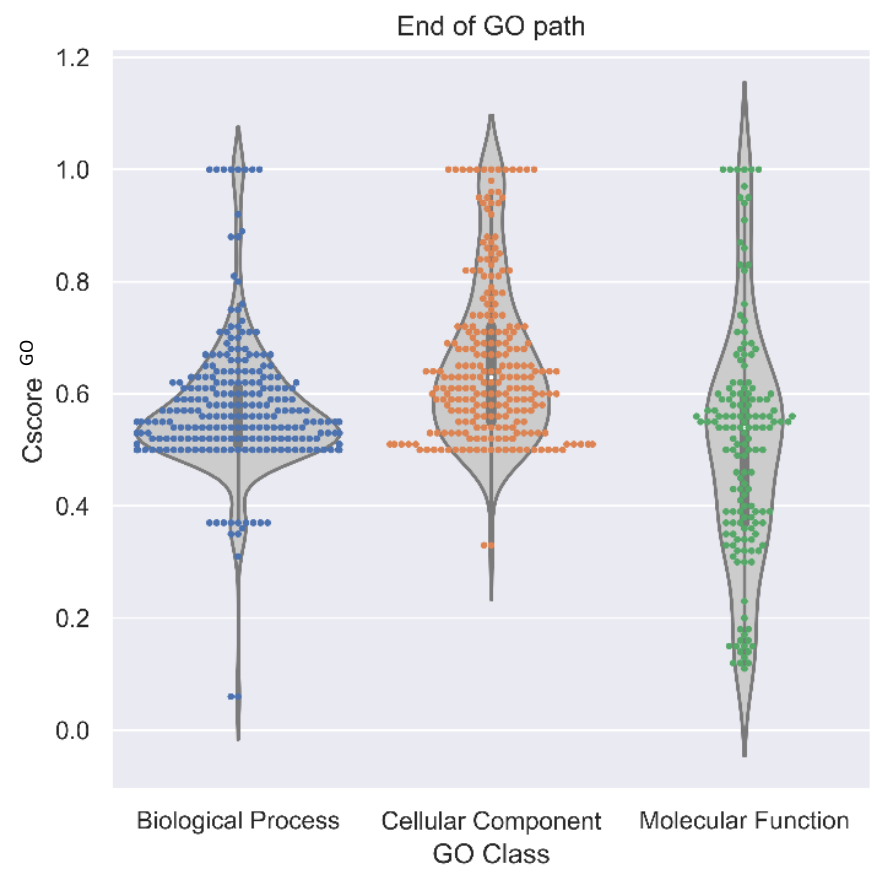


Figure S4. Western blot analysis using 293T cells transfected with DDK-tagged plasmid DNA for three indicated uPE1s: CCDC90B (A), C11orf52 (B) and SMAP (C). Data of western blot analysis with antiCCDC90B(A, upper), anti-C11orf52 (B, upper) anti-SMAP (C, upper), respectively, showed the expression of each uPE1 protein compared to control vector transfection. DDK is detected only in cells expressing CCDC90B (A, bottom), C11orf52 (B, bottom), and SMAP (C, bottom).

A

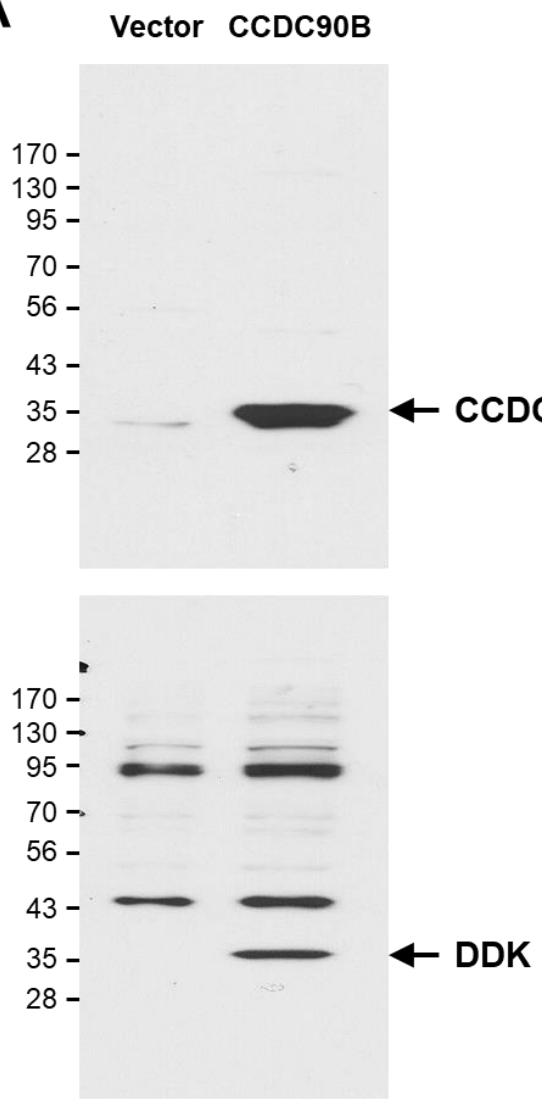

B
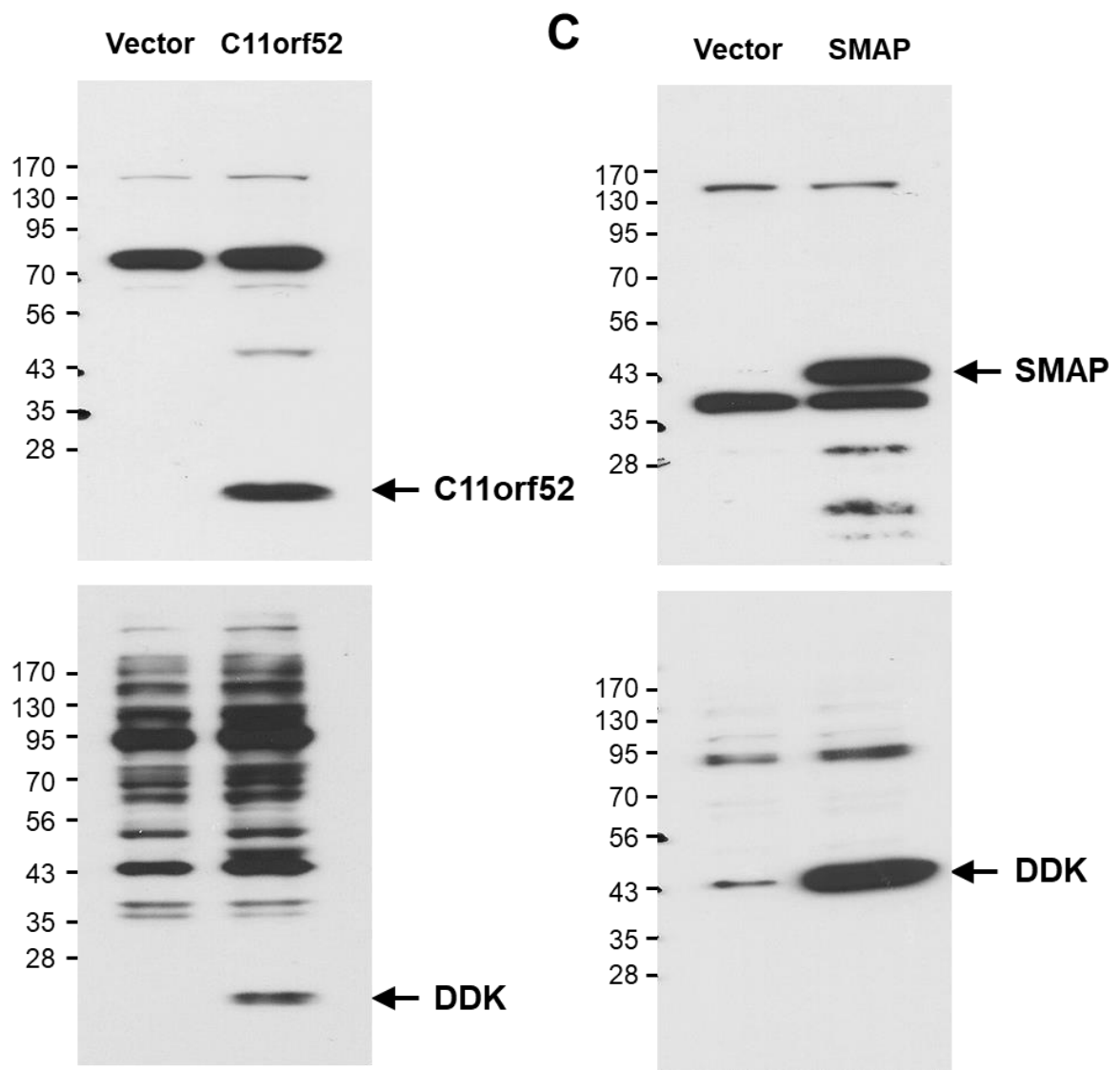


\section{Supplementary Methods \\ Transient Expression (DNA transfection)}

293T cell was purchased from ATCC and cultured in DMEM (Hyclone) suppled with 10\% FBS (Thermo Fisher Scientific) and 1\% Antibiotic-Antimycotic (Thermo Fisher Scientific) at $37^{\circ} \mathrm{C}$ with $5 \% \mathrm{CO} 2$. The number of $3 \times 105$ 293T cells were seeded in 6 well plates and transfections were performed at 70\% confluency. Transfection was performed using Lipofectamine 2000 (Life Technologies) with $0.5 \mu \mathrm{g}$ of plasmid DNA diluted in Opti-MEM. In this experiment, following plasmid DNAs were used: pCMV6-Entry vector, CCDC90B/myc-DDK vector, C11orf52/myc-DDK vector, and SMAP/myc-DDK vector (Origene). After 20minutes incubation at room temperature, the $500 \mu \ell$ of DNA-lipid complex was added to the cells. After incubation for 4 hours at $37^{\circ} \mathrm{C}$ with $5 \% \mathrm{CO}$, the DNA and lipid mixture was changed with high-glucose DMEM suppled with $10 \% \mathrm{FBS}$ and $1 \%$ Antibiotic-Antimycotic. After 2 days incubation at $37^{\circ} \mathrm{C}$ with $5 \% \mathrm{CO} 2$, cells were used to further experiment.

\section{Immunoblotting (Western Blot analysis)}

In order to check transfection efficiency, transfected cells were lysed in RIPA lysis buffer (Biosesang) containing $1 \mathrm{mM}$ phenylmethanesulfonyl fluoride (PMSF), $1 \mathrm{X}$ phosphatase and protease inhibitor cocktail (Sigma), and 0.5M EDTA (Thermo Fisher Scientific). After mechanical dissociation, protein was quantified using a BCA kit. Protein samples were separated by $420 \%$ SDS-PAGE gel (Invitrogen) and transferred to polyvinylidene difluoride (PVDF) membranes. After blocking with $5 \%$ fat-free milk for 1 hour, membrane was probed with following antibodies at $4^{\circ} \mathrm{C}$ overnight: Anti-CCDC90B (Sigma Aldrich, HPA011130, 1: 500), Anti-C11orf52 (Sigma Aldrich, HPA038387, 1:500), Anti-SMAP (Bethyl Laboratories, A304-687A, 1:5000), AntiDDK (Invitrogen, 1:1000),). Rinsed with TBST buffer, the membrane was incubated with antirabbit IgG-HRP antibody, and anti-mouse IgG-HRP (Sigma Aldrich, 1:5000) for 1hour. Immunodetection was carried out with ECL western blotting substrate (GE Healthcare).

\section{Immunofluorescence (IF) staining}

Transfected cells were plated on glass chamber slide coated with $1 \mathrm{mg} / \mathrm{ml}$ Poly-L-Lysine. After 2 days incubation at $37{ }^{\circ} \mathrm{C}$ with $5 \% \mathrm{CO} 2$, cells were fixed and permeabilized with $4 \%$ paraformaldehyde and $100 \%$ ethanol for 15 min and blocked with 1\% BSA for 1 hour at room temperature. Then, the cells were probed with primary antibody for 2 hour and secondary antibody for 1 hour at room temperature. Following antibodies were used: Anti-CCDC90B (Sigma Aldrich; 1:50), Anti-C11orf52 (Sigma Aldrich; 1:50), Anti-SMAP (Bethyl Laboratories; 1:500), and goat anti-rabbit Alexa Fluor 488 (Invitrogen, 1: 200). Hoechst 33342 (Sigma Aldrich, 1:700) and Phalloidin-Alexa568 (Invitrogen, A12380, 1:400) representing nucleus and F-actin, were incubated for $10 \mathrm{~min}$ and 1 hour at room temperature, respectively. Mitotracker (Invitrogen, M7512, 300nM) representing mitochondria was incubated for $30 \mathrm{~min}$ at $37^{\circ} \mathrm{C}$ with $5 \%$ CO2 before fixation. Images were captured using confocal microscopy LSM 880 Airyscan (ZEISS). 